\title{
Thyroid, uncommon metastatic site for renal cell carcinoma - practical challenges
}

\begin{abstract}
Renal cell carcinoma (RCC) is notorious for its uncommon metastatic location. We present a rare case of goiter as a RCC metastatic site. As case particularities, besides the goiter that made practically impossible the ultrasound diagnosis of the metastasis, this seemed to be correlated with an unexplained high level of alkaline phosphatase (AP). AP persisted from the time of kidney cancer diagnosis, after nephrectomy and reached a normal value only after thyroidectomy. Currently, there are no guidelines to indicate FDG-PET in RCC patients. Thereby, we will present the challenging ultrasound diagnosis together with case management particularities.
\end{abstract}

Keywords: kidney cancer, thyroid, distant metastases, ultrasound
Volume II Issue 3 - 2020

\author{
Roxana Maria Pintican, ${ }^{1,2}$ Mihaela Muresan,' \\ Angelica Chiorean, ${ }^{2,3}$ Vasile Bintintan, ${ }^{1,2}$ Vlad \\ Bura,' Magdalena Duma ${ }^{2-4}$ \\ 'County Emergency Hospital, Romania \\ 2“Iuliu Hatieganu” University of Medicine and Pharmacy, \\ Romania \\ ${ }^{3}$ Medimages Clinic, Constantin Brancusi Street, Romania \\ ${ }^{4}$ Micromedica Clinic, Petru Rares Street, Romania
}

\begin{abstract}
Correspondence: Roxana Maria Pintican, County Emergency Hospital, Clinicilor Street, nr 3-5, Cluj-Napoca, "Iuliu Hatieganu" University of Medicine and Pharmacy,Victor Babes Street, nr 8, Cluj-Napoca, Romania, Tel 0040729/21 I22,

Email roxana.pintican@gmail.com
\end{abstract}

Received: June 05, 2020 | Published: June 23, 2020

\section{Introduction}

Renal cell carcinoma (RCC) is the most common type of kidney cancer in adults and it accounts for more than $3 \%$ of adult malignancies. ${ }^{1}$ It is well known for its unpredictable behavior regarding distant metastases after long disease-free intervals. Aside the common sites of metastatic spread, rare and very rare locations exist, such as the thyroid gland. ${ }^{2}$

\section{Case}

We present the case of a $60 \mathrm{y}$ old female smoker with new onset dyspnea. Patient's medical history includes a left nephrectomy for stage I RCC, grade 3, clear cell type (2016) and a macro-nodular goiter (2010). The latter was followed up over the years, despite the endocrinologist indication for surgery.

The thyroid ultrasound showed the same profusely inhomogeneous macro-nodular goiter as was in 2010 , with a dominant left noduleFigure 1 upper images. The heterogeneous thyroid plunged within the antero-superior mediastinum. No suspicious lateral-cervical lymphnodes were noted. Particularly, an unexplained high level of alkaline phosphatase $(\mathrm{AP}=321 \mathrm{mg} / \mathrm{dl})$ persisted from the time of kidney cancer diagnosis and after nephrectomy.

The patient agreed to a total thyroidectomy. Intraoperatively, the surgeon found stiff subcentimetric lymphnodes located posteriorly to the goiter and thus a preemptive extended cervical lymphadenectomy was performed. Post-surgery, the pathology analysis revealed clear cytoplasmic cell groups disposed among the thyroid follicles. The cells were positive for CD10 antibody implying a renal origin. The TTF-1 (thyroid transcription factor 1), calcitonin, galectin 3 and CK 19 negativity, excluded a thyroid origin of the cells - Figure 1 lower image. All excised lymphnodes were free of metastatic disease. The final diagnosis was RCC metastasis within the thyroid, with no signs of malignant transformation of the nodular goiter.
Post operatively, the AP reached a normal level (bellow $200 \mathrm{mg}$ / dl). Given the unusual metastatic spread, the oncologist thought prudent to supplement patient investigations with a PET-CT to make sure that the rest of the body was indeed free of secondary lesions. The PET-CT scan showed high SUV uptake at the right parapharyngeal space. An ear-nose-throat exam and needle biopsy were performed; the results revealed inflammation, most probably related to smoking.

The patient is currently under active monitoring with no adjuvant oncologic therapy. The 6 months post-surgery follow up including cervical and thorax CT plus abdominal and pelvic MRI were negative for signs of relapse.

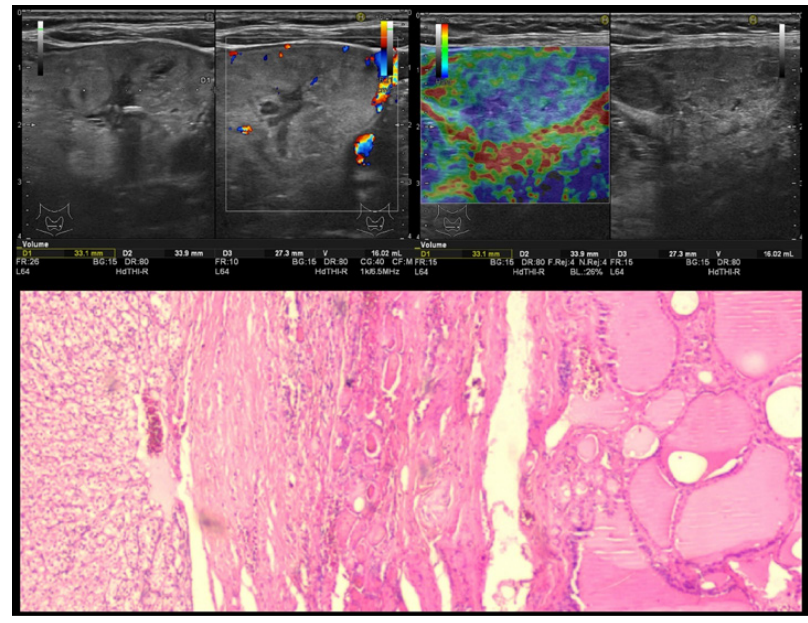

Figure I Upper images-thyroid ultrasound: Heterogeneous appearance with cystic areas and peripheral vascularity. Note the nonspecific, mosaic-like elastographic appearance (on the right), difficult to grade in Asteria scoring system. Lower image - Histology sample: Hematoxylin eosin stain (HE) $4 x$ magnification, showing renal cell carcinoma in the left and thyroid follicles in the right (a) 


\section{Discussion}

Even though metastatic disease is a sequential process, RCC defies us by its unpredictable behavior. In our case the thyroid metastasis if detected before surgery would have meant a stage IV renal cell carcinoma and according to literature only $8 \%$ chances to survive in the next 5 years.

Concerning other paraclinical biomarkers for metastatic RCC, it is known that recurrent or persistent serum AP elevation after radical nephrectomy suggests distant metastasis or residual tumor. In our case the persistent increased value of AP after nephrectomy in spite the apparent lack of distant spread or recurrence confounded the clinicians. Normal values were reached only post-thyroidectomy.

Despite the rich vascular supply of the thyroid, only $2-3 \%$ of its malignancies are represented by a metastatic nodule. The lower frequency of metastases can be explained by the high iodine and oxygen concentrations, ${ }^{3}$ that is why patients with adenomatous goiter who have lower iodine levels may be more exposed to developing thyroid metastases.

Regarding the ultrasound features in patients without previous thyroid pathology, these distant carcinomas were described as solitary, irregular, and solid nodule with no calcifications. ${ }^{4}$ In our case, a previously macronodular background made it practically impossible to identify any sign of metastatic disease.

The use of fine needle aspiration biopsy might be helpful, however it may yield false-negative results if a benign nodule is sampled, making the diagnostic of a metastatic nodule in a patient with goiter cumbersome. On the topic of systemic therapy for metastatic RCC, the latest kidney cancer guidelines do not recommend drugs like Sunitinib for patients who underwent a metastasectomy. In addition, thyroid metastases are not among the ones that are amenable for the systemic therapy, like lung, bone and brain metastases. ${ }^{5}$

The FDG-PET examination is not routinely recommended for diagnosis or staging RCC patients. ${ }^{5}$ It is difficult to speculate retrospectively that a PET scan would have revealed the thyroid metastasis but perhaps an unexplained elevated AP value in nephrectomized patients for RCC, may be a reason to consider adding an FDG examination to amend the investigative protocol (further studies are needed).

\section{Conclusion}

The metastatic pathways of RCC are not fully understood, especially for uncommon locations. Radiologists need to keep in mind the possibility of goiter as a site for metastatic spread, although the ultrasound features are not always characteristic. A new onset or a change in symptoms might be the only clue that something unfortunate is happening at the molecular level, with a huge impact on prognosis and overall survival. An unexplained elevated AP value in RCC nephrectomised patients may follow an undiagnosed metastasis. In this case, FDG examination may play an important role in their clinical management (further studies are needed).

\section{Acknowledgments}

None.

\section{Conflicts of interest}

The authors declare there is no conflict of interest.

\section{Funding}

None.

\section{References}

1. Beutner U, Leowardi C, Bork U, et al. Survival after renal cell carcinoma metastasis to the thyroid: single center experience and systematic review of the literature. Thyroid. 2015;25(3):314-324.

2. Sountoulides P, Metaxa L, Cindolo L. Atypical presentations and rare metastatic sites of renal cell carcinoma: A review of case reports. $J$ Med Case Rep. 2011;5:429.

3. Cilengir AH, Kalayci TO, Duygulu G, et al. Metastasis of renal clear cell carcinoma to thyroid gland mimicking adenomatous goiter. Polish J Radiol. 2016;81:618-621.

4. Kobayashi K, Hirokawa M, Yabuta T, et al. Metastatic carcinoma to the thyroid gland from renal cell carcinoma: Role of ultrasonography in preoperative diagnosis. Thyroid Res. 2015;8(1).

5. Escudier B, Porta C, Schmidinger M, et al. Renal cell carcinoma: ESMO Clinical Practice Guidelines for diagnosis, treatment and follow-up. Ann Oncol. 2019;30(5):706-720. 